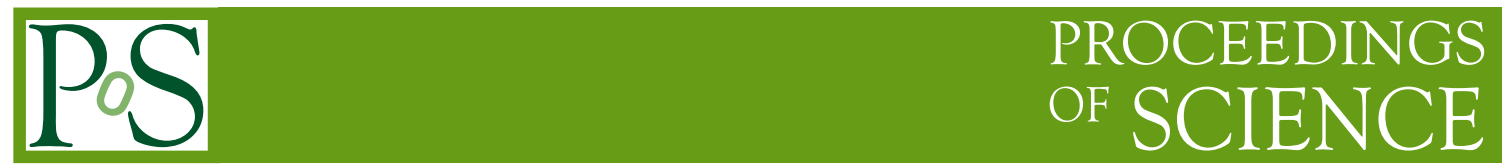

\title{
Dust in Star Forming Regions - A Review
}

\section{Dmitri Wiebe*}

Institute of Astronomy of the Russian Academy of Sciences, Moscow, Russia

E-mail: dwiebe@inasan.ru

\begin{abstract}
Space dust is a major astronomical factor, which affects most observations in UV, optical, infrared, submillimeter, etc. Most often it is an obstacle, preventing us from getting undistorted information on various astronomical objects. Dust in star-forming regions is different. It is not only an obstacle but also an important tracer of physical conditions in these regions, a catalyst for interstellar chemistry, and a progenitor for planetary systems. In order to clarify all these various roles, we need to know properties of space dust and how they may change on different stages of the star formation process.
\end{abstract}

Frontier Research in Astrophysics - II

23-28 May 2016

Mondello (Palermo), Italy

\footnotetext{
* Speaker.
} 


\section{Multitude of Dust Models}

Historically, the easiest way to notice the presence of dust in the interstellar medium was related to its property of absorbing starlight. While the possible existence of interstellar absorbing substance has been mentioned back in 1847 by F. G. W. Struve in his "Studies in Stellar Astronomy", the ultimate discovery of interstellar dust is often attributed to the paper of Trumpler, published in 1930 [1]. He has noticed that remote open star clusters appear to be dimmer and redder than similar clusters located closer to the Sun. He has related this effect to the presence of tiny dust particles mostly concentrated to the plane of the Milky Way.

A more sophisticated procedure to infer interstellar dust properties has been introduced in 1936 by Rudnick [2]. This procedure is sometimes called a pair-matching and consists of comparing spectra of two stars of the same spectral type. One of these stars should be relatively nearby, thus, experiencing less reddening. The second star should be more reddened, being located further away or, probably, residing behind a dense cloud of obscuring material. As the spectral types are the same, we expect that the real spectra are identical, and all the observed differences between them are caused by the interstellar extinction. The difference between the "true" spectrum and the observed spectrum is known as the interstellar extinction curve. The first task that needs to be solved by a good dust model is the explanation for this curve, that is, for the dependence of absorption on the wavelength of radiation.

As it turned out, two key features of the interstellar extinction curve are located outside of the visible band, so they have only been discovered after it had become possible to perform observations in ultraviolet (UV) and infrared (IR) bands.

The first of these features is the so-called UV bump at the wavelength of about $2200 \AA$. Its existence has been clearly demonstrated by Stecher [3], using rocket-based UV observations, and attributed to graphite by Stecher and Donn [4]. The second feature, or rather a pair of features, is observed in the infrared and is assumed to be related to silicate dust. Specifically, a feature at $\sim 10 \mu \mathrm{m}$ is believed to be related to stretching of Si-O bonds, while a feature at $\sim 18 \mu \mathrm{m}$ is attributed to O-Si-O bending. Unlike the UV bump, the silicate features can be observed both in absorption and in emission. Actually, the $10 \mu \mathrm{m}$ feature has first been observed as an emission peak in spectra of some cool stars [5].

The overall shape of the interstellar extinction curve has become a basis for the famous MRN model [6], according to which the space dust is a mix of graphite and silicate grains with a power law size distribution

$$
\frac{d n}{d a} \propto a^{-3.5}
$$

and size limits from $0.005 \mu \mathrm{m}$ to $1 \mu \mathrm{m}$ for graphite grains and about $0.025-0.25 \mu \mathrm{m}$ for silicate materials.

The MRN model has become (and still is) extremely popular due to its simplicity, but somewhat later it has been shown that while it is successful in explaining dust absorption, it fails when it comes to dust emission. After IRAS it became obvious that the MRN model is not able to reproduce strong dust emission in the mid-IR range, that is, at wavelengths of about $10-30 \mu \mathrm{m}$ [7]. This excess emission stressed the necessity to consider an additional dust population coined very small grains (VSG). They are different from larger grains in their heating regime. The temperature 
of large interstellar grains (with the total thermal energy much greater than the energy of a single absorbed photon) is defined by the equilibrium between absorption of diffuse stellar radiation and dust own thermal emission. For the "MRN" grains this equilibrium temperature is quite low, only of the order of $15 \mathrm{~K}$. Thus, they cannot make any significant contribution in mid-IR emission. On the other hand, smaller grains (with the total thermal energy lower than the energy of a single absorbed photon) experience transient heating up to a temperature, which can be much higher than the equilibrium temperature, upon the single photon absorption. These stochastic heating events in the grain ensemble produce observed excess mid-IR emission (see e.g. [8, 9]).

Yet another grain component reveals itself through emission bands in the near infrared. The first so called unidentified infrared band (UIB) at $11.2 \mu \mathrm{m}$ has first been observed by Gillett et al. [10] in some planetary nebulae. Later it turned out that there are other UIBs at many wavelengths between 3 and $20 \mu \mathrm{m}$, and these emission bands are truly ubiquitous and present in many different objects, including star-forming regions. Bands instead of a pure continuum apparently hint at some particles that are even smaller than very small grains. After Leger and Puget [11] and Allamandola et al. [12], the origin of the UIBs is generally assumed to be related to polycyclic aromatic hydrocarbons $(\mathrm{PAH})$, which are planar macromolecules, consisting of some number of benzene rings. It must be noted that despite this generally adopted attribution the infrared bands still stay unidentified as all the attempts to assign the observed bands to a certain PAH variety has so far been unsuccessful. Even more so, it has been demonstrated by Rosenberg et al. [13] that one needs at least 30 different PAH molecules to reproduce the observed properties of the UIBs, but it does not really matter, which specific PAH molecules are included in this mix. Also, it is possible that the aromatic compounds do not present in the ISM as "pure" PAH particles but rather are incorporated in more complicated structures (see e.g. [14]).

All these new developments have been incorporated in another popular dust model, published by Weingartner and Draine [15]. According to this model, the space dust consists of a silicate component and a carbonaceous component. The former is basically similar in its properties to the analogous component of the MRN model. The latter along with a "conventional" MRN-like dust includes also VSGs with a typical size of about $50 \AA$ and PAHs with a typical size of a few $\AA$.

It has been shown many times that the model developed Weingartner and Draine is quite successful in describing dust observations both in our Galaxy and in other galaxies. However, this does not imply that the story of dust models is told to the very end. The combination of silicate grains, carbonaceous grains, and PAHs, proposed in [15], is by no means unique. A good illustration of this statement has been provided by Zubko et al. [16]. These authors have tried to fit absorption constraints, emission constraints, and abundance constraints simultaneously, using various combinations of several dust materials, including silicates, graphite, amorphous carbon, PAHs, and composite particles. It has been shown that while no unique interstellar dust model is able to fit simultaneously all the available constraints, there are several classes of acceptable interstellar dust models, including those, for example, which do not require the presence of graphite particles at all.

More information on our current understanding of space dust and its history can be found in reviews and books $[17,18,19,20]$. 


\section{Dust as a Tracer of Physical Conditions in Star-Forming Regions}

Dust is the most important astrophysical topic ever. Suffice it to say that in the ADS citation count the most cited astrophysical paper is the paper by Schlegel et al. [21], which describes maps of Galactic dust infrared emission. The reason is simple. Dust is an obstacle for almost any observations performed in UV, visible, infrared, submillimeter, and millimeter bands, including cosmologically important observations of the cosmic microwave background. Obviously, in order to clean these observations from dust contribution one has to know both dust properties and its spatial distribution.

But in observations of star-forming regions dust is no more just an obstacle, but an important tracer of physical conditions and factors, which accompany or even provoke and control the star formation process. Also, dust is not a mere witness of this process, but an important factor itself. It is a major energy sink during the protostellar collapse, a curtain that protects molecules in dense clouds from being dissociated by UV radiation, a catalyst for many important chemical reactions, including formation of molecular hydrogen. Finally, dust is a raw material for planetary systems.

\subsection{Density and Temperature}

Star formation occurs in molecular clouds. The irony of the Universe is that the major component of molecular matter, $\mathrm{H}_{2}$ molecule, cannot be observed directly due to the lack of transitions, which would be effectively excited in cold molecular clouds. Thus, in our studies of the star formation process we have to rely on observations of admixture components, namely, dust and molecules. At the first sight, molecules seem to be more useful as they are a source of spectral lines, which means we can harness the full power of spectral analysis for star formation studies. Molecules can be used to infer gas density and temperature, velocity field, magnetic field, and radiation field. Two difficulties are that 1) the modeling of radiation transfer in molecular lines is far from being straightforward and 2) molecules are not well mixed with the bulk of the gas. Some molecules are more abundant in dense clumps and cores, while others are present mainly at the cloud periphery. One has to understand all these details in order to interpret line observations properly.

Dust in this respect appears to be simpler. Emission of at least larger grains can be treated as black body emission at equilibrium dust temperature, which greatly simplifies the solution of the radiation transfer problem, especially, in the optically thin case. Also, it is generally assumed that dust is well mixed with gas (but see [22] for a different view). So, it may seem that observing dust does allow inferring more global properties of interstellar matter.

A well-known example of using dust as a proxy for the overall gas distribution has been published by Alves et al. [23]. These authors have used observations in visible and near-IR ranges to study extinction along numerous lines of sight through the dark globule B68. This object is a very popular target for various observations as it is relatively nearby (so that there are no foreground stars) and well isolated from other dark clouds. Measuring extinction for background star and using these data to estimate total column density, Alves et al. have found that matter distribution in B68 closely resembles that of a so-called Bonnor-Ebert sphere, which is a gas sphere in hydrostatic equilibrium, stabilized by external pressure. This resemblance has been considered as an evidence in favor of the "standard" model of star formation, in which molecular clouds are long-living entities, supported against collapse by magnetic field. However, this conclusion has later been shaken 
by results of numerical modeling. Ballesteros-Paredes et al. [24] have modeled the formation of dense core in a turbulent molecular clouds and found that more than a half of these cores have density profiles which would be observationally similar to those of Bonnor-Ebert spheres, while in fact the cores are neither stable, nor static.

Another case against straightforward interpretation of dust observations in terms of the core stability has been presented by Kirk et al. [25]. Unlike Alves et al. [23], they have used emission data to restore density profiles. It was found that observed profiles can only be fitted by the BonnorEbert profile under the assumption of unrealistically high dust temperature.

This does not necessarily mean that the Bonnor-Ebert stability criterion is useless. For example, Kandori et al. [26] have used dust extinction measurements in 10 Bok globules to find their Bonnor-Ebert dimensionless radii $\xi_{\max }$ and to relate them to the critical value $\xi_{\text {crit }}=6.5$, separating stable and unstable configuration. It was found that starless cores may have both subcritical and supercritical $\xi_{\max }$ values, but all the star-forming globules are unstable in terms of the Bonnor-Ebert analysis. So, there is some meaning in this kind of analysis, but nevertheless one has to be very careful, interpreting dust observations in terms of density and core stability.

The situation becomes more certain when data for many wavelengths across the spectrum from infrared band to millimeter band are available. E.g., Launhardt et al. [27] have utilized multi-wavelength Herschel observations to derive surface density and temperature in a number of molecular cloud cores. It was shown that the thermal structure of the cores is dominated by external heating, while internal sources (protostars), if there are any, only affect the innermost regions of the cores.

The most recent development in studying interstellar matter distribution in star-forming regions using dust observations is related not to individual cores but to a large-scale structure of molecular clouds. Survey observations performed with Herschel show that molecular clouds have filamentary structure, with the star formation proceeding along these filaments. While filaments in molecular clouds have been known long before Herschel, it is now clear that they are ubiquitous, and this is sometimes called no less than the new paradigm of star formation. In this paradigm, first filaments form in molecular clouds, and later their fragmentation gives rise to newly-born stars and star clusters. A signature of a filament growth can presumably be seen in thinner perpendicular "striations" attached to the main filament [28]. Unfortunately, numerical models do not allow isolating the major factor (turbulence, magnetic field) responsible for the formation of filaments [29].

\subsection{Magnetic Field}

Magnetic field is definitely an important player in the star formation process but its specific role is still a matter of a debate. Dust observations have long been seen as a tool for determining the magnetic field structure in molecular clouds through polarimetric observations.

Polarization of starlight has been discovered in 1949 [30, 31] and was almost immediately recognized as arising from absorption and scattering of starlight by elongated dust grains that are partially aligned by the magnetic field [32]. Thus, dust observations have become a tool to determine the structure of the galactic magnetic field at various scales. The most probable orientation of dust grains is such that their longer axes are perpendicular to the magnetic field direction. Starlight traversing the dusty medium attains polarization parallel to the magnetic field projection onto the 
plane of the sky (POS). Proper dust thermal emission is partially polarized in the direction perpendicular to the POS magnetic field. (It must be kept in mind that dust polarization is only sensitive to the component of the magnetic field, perpendicular to the line of sight.)

Obviously, polarization of starlight and polarization of dust thermal emission are observed in very different circumstances, with the former corresponding to lines of sight with low column density and the latter corresponding to lines of sight with high column density. However, when it is possible to compare inferences from these two kinds of observations, they seem to draw a consistent picture [33]. In this picture, the large-scale magnetic field in star-forming regions is either parallel or perpendicular to filaments, proving a certain connection between the magnetic field structure and the overall matter distribution [34]. There are indications that in the diffuse medium the magnetic field is mostly parallel to elongated cloud structures (filaments), while in the denser medium the magnetic field can be both parallel and perpendicular to these structures [35]. This can trace a phase of a filament growth, when matter flows into the filament along the magnetic field lines.

When we consider smaller scales, dust polarimetry may become less useful. One of the evidences in favor of dust-related polarization of starlight is that polarization degree $p$ grows with extinction. That is, we should have more polarization when we have more dust on the line of sight. However, in 1990s it was found that this trend only appears when we observe stars located behind the general interstellar medium, that is, when large amount of intervening dust is caused by large distance to the star. If the star is located behind a dense cloud, that is, the large amount of dust is caused by high density on the line of sight, the polarization does not grow with extinction up to rather high extinction values $\left(A_{V} \sim 10^{\mathrm{m}}\right)[36,37,38]$.

The similar effect is also observed in polarization of dust thermal emission. In the case of starlight polarization we expect $p$ to grow with dust column density, but in dense clouds $p$ actually stays nearly constant. In the case of polarized dust thermal emission we expect $p$ to be independent on dust column density but it actually decreases. This effect is called a "polarization hole' (see e.g. $[35,39])$, as it is mostly observed in centrally peaked sources and appears to be quite wide-spread (albeit not universal).

Various explanations have been proposed for the "polarization hole" effect, including dust grain spherization, less efficient alignment mechanisms at higher density, optical depth effects (see discussion in Wiebe \& Watson [40]). One of the plausible explanations relates less polarization at higher density to more irregular structure of the magnetic field in dense clouds [41]. Indeed, the polarization hole is present in the Kleinmann-Low Nebula in OMC-1, when it is observed with low angular resolution at $100 \mu \mathrm{m}$ [42], and disappears, when observed at higher resolution and larger wavelength [43].

Another example of the apparently twisted magnetic field structure has been presented recently by Tang et al. [44]. They have observed the massive star-forming region W51 North at various angular resolutions and found more and more complicated magnetic field morphology as they moved from the scale of the whole cloud to the scale of individual objects, with an hour-glass structure, expected for protostars with disks, at the smallest considered scale. Surprisingly, the polarization hole effect is seen at all the scales, with almost the same maximum polarization degree from the entire cloud to individual cores. This apparently indicates that more than one factor probably leads to less polarization at higher intensity. 


\section{Dust Evolution in Star-Forming Regions}

Yet another complication in using dust as a tracer of the star formation process is related to the fact that during this process dust probably does not stay the same.

\subsection{Dust Formation}

It was long thought that space dust is mostly produced in extended envelopes of evolved stars [45]. However, there is a long way from these stars to molecular clouds, and there are many processes on this way, which effectively destroy dust grains. The major destruction factor, supernova explosions, is so effective that it may completely destroy all the dust synthesized in evolved stars in our Galaxy [46]. While efficiency of dust destruction in supernova remnants may be overestimated, and supernovae may not only destroy, but also produce dust, one still needs to consider other dust sources in our Galaxy and in extragalactic sources. One possible answer to the question on alternative dust sources is that dust may grow or even form in the ISM and molecular clouds themselves [47].

Evidences for bigger dust grains in dense molecular medium than in the general ISM are actually quite numerous. The simplest way to check if this is indeed the case is to look at the parameter $R_{V}=A_{V} / E(B-V)$. Its standard value for the Milky Way galaxy is 3.1, but $R_{V}$ variations in different environments can be quite significant. Large $R_{V}$ values correspond to greater average grain size, and they are typically found in star-forming regions. For example, Kandori et al. [48] have found that in the non-star-forming part of the cloud L1251 $R_{V}$ has a typical interstellar value, while in the denser part of the cloud, already enveloped in star formation, $R_{V}=6.5$. A similar relation between $R_{V}$ and extinction (the larger $A_{V}$, that is, density, the larger $R_{V}$ ) has been found by Allen et al. [49]. Growth of $R_{V}$ toward the center of the globule CB107 has been noticed by Campeggio et al. [50].

Another evidence in favor of grain growth in molecular clouds is the so-called "coreshine", faint glow at near-IR Spitzer bands observed in some dark clouds (see [51] and references therein). This glow is believed to be the starlight scattered by dust grains, which in some clouds should have an upper size limit greater than $1.5 \mu \mathrm{m}$.

An open question is whether this growth proceeds through coagulation (constant dust mass) or accretion from the gas (growing dust mass). Evidence in favor of the second option has been presented by Voshchinnikov and Henning [52]. They have related density (parameterized as reddening per unit length) to the heavy element depletion and found that the depletion grows with density, which is indicative of grain growth due to accretion of iron, magnesium, and silicon atoms from gas to dust.

\subsection{Dust Destruction}

Speaking of dust destruction in star-forming regions, I will only consider the specific problem of dust evolution in HII regions and complexes. One of the dust-related discoveries made with Spitzer telescope was the discovery of so-called infrared bubbles. More accurately, infrared ring nebulae have been known for a long time, however, only after Spitzer it has become clear that they are, first, very numerous [53, 54], second, mostly connected to HII regions around young massive stars [55]. 
These bubbles have a very distinctive morphology. An ionized hydrogen region is surrounded by a closed or broken ring of $8 \mu \mathrm{m}$ emission. Coincident with this ring are also rings of emission at $24 \mu \mathrm{m}, 70 \mu \mathrm{m}$ and longer wavelengths. The inner part of the region (enclosed in the $8 \mu \mathrm{m}$ ring) is the source of bright emission at $24 \mu \mathrm{m}$. While the origin of $24 \mu \mathrm{m}$ emission is still unclear $[9,55]$, aromatic compounds (PAHs) seem to be the only reliable source of emission at $8 \mu \mathrm{m}$. Correspondingly, the absence of $8 \mu \mathrm{m}$ emission in the inner part of the region reflects the absence of PAHs. Pavlyuchenkov et al. [56] have shown that this can be explained if we assume that all the PAH molecules are destroyed by radiation from a central star on a timescale of about $3-30$. $10^{6} \mathrm{yrs} / G$, where $G$ is the intensity of the local radiation field in units of the average interstellar radiation field.

On the scale of whole HII complexes in extragalactic star-forming regions this problem has been studied by Wiebe et al. [57] and Khramtsova et al. [58]. The surprising result of this study was that despite all the evidences that PAHs should be destroyed by UV radiation their mass fraction actually grows with age in star-forming complexes of low metallicity, as if they were destroyed less efficiently than larger grains. The expected trend, that is, PAH mass fraction decreasing with age, has only been observed at solar or higher metallicities. One way to explain this result is to assume that PAHs (or some more general aromatic material) are simultaneously destroyed by UV photons and produced due to aromatization and destruction of larger carbonaceous grains [59].

This means that dust evolution in star-forming regions is not bound exclusively to mere formation and destruction processes. One also has to take into account possible dust restructuring from one sort of material to another. We have initiated our own modeling of this kind. The initial results are presented in $[60,61]$.

\section{Conclusions}

We know for sure that space dust mainly consists of silicate and carbonaceous components. The relative contribution of various dust constituents is still unclear, but it almost definitely varies spatially and changes with time. Processing in star-forming regions can be an important factor in dust destruction, formation, and transformation. In order to interpret observations of star-forming regions properly, we need to pay a due attention to all these factors.

This study has been supported by the NSh-9951.2016.2 grant and the RFBR grant 14-0200604 .

\section{References}

[1] R. Trumpler, Absorption of Light in the Galactic System, PASP 42, 214 (1930).

[2] J. Rudnick, On the Reddening in B-Type Stars, ApJ 83, 394 (1936).

[3] T.P. Stecher, Interstellar Extinction in the Ultraviolet, ApJ 142, 1683 (1965).

[4] T.P. Stecher, B. Donn, On Graphite and Interstellar Extinction, ApJ 142, 1681 (1965).

[5] F.C. Gillett, F.J. Low, W.A. Stein, Stellar Spectra from 2.8 to 14 Microns, ApJ 154, 677 (1968).

[6] J.S. Mathis, W. Rumpl; K.H. Nordsieck, The Size Distribution of Interstellar Grains, ApJ 217, 425 (1977). 
[7] J.L. Weiland, L. Blitz, E. Dwek, M.G. Hauser, L. Magnani, L.J. Rickard, Infrared Cirrus and High-Latitude Molecular Clouds, ApJ 306, L101 (1986).

[8] B.T. Draine, A. Li, Infrared Emission from Interstellar Dust. IV. The Silicate-Graphite-PAH Model in the Post-Spitzer Era, ApJ 657, 810 (2007).

[9] Ya.N. Pavlyuchenkov, D.S. Wiebe, V.V. Akimkin, M.S. Khramtsova, Th. Henning, Stochastic Grain Heating and Mid-Infrared Emission in Protostellar Cores, MNRAS 421, 2430 (2012).

[10] F.C. Gillett, W.J. Forrest, K.M. Merrill, 8-13-micron Spectra of NGC 7027, BD +30 3639, and NGC 6572, ApJ 183, 87 (1973).

[11] A. Leger, J.L. Puget, Identification of the "Unidentified” IR Emission Features of Interstellar Dust? $A \& A$ 137, L5 (1984).

[12] L.J. Allamandola, A.G.G.M. Tielens, J.R. Barker, Polycyclic Aromatic Hydrocarbons and the Unidentified Infrared Emission Bands: Auto Exhaust along the Milky Way, ApJ 290, L25 (1985).

[13] M.J.F. Rosenberg, O. Berné, Ch. Boersma, Random Mixtures of Polycyclic Aromatic Hydrocarbon Spectra Match Interstellar Infrared Emission, A\&A 566, L4 (2014).

[14] A.P. Jones, Variations on a Theme - the Evolution of Hydrocarbon Solids. I. Compositional and Spectral Modelling - the eRCN and DG Models, A\&A 540, A1 (2012).

[15] J.C. Weingartner, B.T. Draine, Dust Grain-Size Distributions and Extinction in the Milky Way, Large Magellanic Cloud, and Small Magellanic Cloud, Apj 548, 296 (2001).

[16] V. Zubko, E. Dwek, R.G. Arendt, Interstellar Dust Models Consistent with Extinction, Emission, and Abundance Constraints, ApJS 152, 211 (2004).

[17] B.T. Draine, Interstellar Dust Grains, ARA\&A 41, 241 (2003).

[18] E. Krügel, The Physics of Interstellar Dust, Institute of Physics, Bristol 2003.

[19] A. Li, Interstellar Grains—the 75th Anniversary, Journal of Physics: Conference Series 6, 229 (2005)

[20] B.T. Draine, Physics of the Interstellar and Intergalactic Medium, Princeton University Press, Princeton 2011.

[21] D.J. Schlegel, D.P. Finkbeiner, M. Davis, Maps of Dust Infrared Emission for Use in Estimation of Reddening and Cosmic Microwave Background Radiation Foregrounds, ApJ 500, 525 (1998).

[22] P. Padoan, L. Cambrésy, M. Juvela, A. Kritsuk, W.D. Langer, M.L. Norman, Can We Trust the Dust? Evidence of Dust Segregation in Molecular Clouds, ApJ 649, 807 (2006).

[23] J. Alves, Ch.J. Lada, E.A. Lada, Internal Structure of a Cold Dark Molecular Cloud Inferred from the Extinction of Background Starlight, Nature 409, 159 (2001).

[24] J. Ballesteros-Paredes, R.S. Klessen, E. Vázquez-Semadeni, Dynamic Cores in Hydrostatic Disguise, ApJ 592, 188 (2003).

[25] J.M. Kirk, D. Ward-Thompson, P. André, The Initial Conditions of Isolated Star Formation - VI. SCUBA Mapping of Pre-Stellar Cores, MNRAS 360, 1506 (2005).

[26] R. Kandori, Y. Nakajima, M. Tamura, K. Tatematsu, Y. Aikawa, T. Naoi, K. Sugitani, H. Nakaya, T. Nagayama, T. Nagata, M. Kurita, D. Kato, C. Nagashima, Sh. Sato, Near-Infrared Imaging Survey of Bok Globules: Density Structure, AJ 130, 2166 (2005). 
[27] R. Launhardt, A.M. Stutz, A. Schmiedeke, Th. Henning, O. Krause, Z. Balog, H. Beuther, S. Birkmann, M. Hennemann, J. Kainulainen, T. Khanzadyan, H. Linz, N. Lippok, M. Nielbock, J. Pitann, S. Ragan, C. Risacher,5, M. Schmalzl, Y.L. Shirley, B. Stecklum, J. Steinacker, J. Tackenberg, The Earliest Phases of Star Formation (EPoS): a Herschel Key Project. The Thermal Structure of Low-Mass Molecular Cloud Cores, A\&A 551, A98 (2013).

[28] P. Palmeirim, Ph. André, J. Kirk, D. Ward-Thompson, D. Arzoumanian, V. Könyves, P. Didelon, N. Schneider, M. Benedettini, S. Bontemps, J. Di Francesco, D. Elia, M. Griffin, M. Hennemann, T. Hill, P.G. Martin, A. Men'shchikov, S. Molinari, F. Motte, Q. Nguyen Luong, D. Nutter, N. Peretto, S. Pezzuto, A. Roy, K.L.J. Rygl, L. Spinoglio, G.L. White, Herschel View of the Taurus B211/3 Filament and Striations: Evidence of Filamentary Growth? A\&A 550, A38 (2013).

[29] Ch. Federrath, On the Universality of Interstellar Filaments: Theory Meets Simulations and Observations, MNRAS 457, 375 (2016).

[30] W.A. Hiltner, Polarization of Radiation from Distant Stars by the Interstellar Medium, Nature 163, 283 (1949).

[31] J.S. Hall, Observations of the Polarized Light from Stars, Science 109, 166 (1949).

[32] L. Davis Jr., J.L. Greenstein, The Polarization of Starlight by Aligned Dust Grains, ApJ 114, 206 (1951).

[33] Planck Collaboration, Planck Intermediate Results. XXI. Comparison of Polarized Thermal Emission from Galactic Dust at $353 \mathrm{GHz}$ with Interstellar Polarization in the Visible, A\&A 576, A106 (2015).

[34] Planck Collaboration, Planck Intermediate Results. XXXV. Probing the Role of the Magnetic Field in the Formation of Structure in Molecular Clouds, A\&A 586, A138 (2016).

[35] J. Malinen, L. Montier, J. Montillaud, M. Juvela, I. Ristorcelli, S.E. Clark, O. Berné, J.-Ph. Bernard, V.-M. Pelkonen, D.C. Collins, Matching Dust Emission Structures and Magnetic Field in High-Latitude Cloud L1642: Comparing Herschel and Planck Maps, MNRAS 460, 1934 (2016).

[36] A.A. Goodman, T.J. Jones, E.A. Lada, Ph.C. Myers, The Structure of Magnetic Fields in Dark Clouds - Infrared Polarimetry in B216-217, ApJ 399, 108 (1992).

[37] A.A. Goodman, T.J. Jones, E.A. Lada, Ph.C. Myers, Does Near-Infrared Polarimetry Reveal the Magnetic Field in Cold Dark Clouds? ApJ 448, 748 (1995).

[38] H.G. Arce, A.A. Goodman, P. Bastien, N. Manset, M. Sumner, The Polarizing Power of the Interstellar Medium in Taurus, ApJL 499, L93 (1998).

[39] Sh.-P. Lai, R.M. Crutcher, J.M. Girart, R. Rao, Interferometric Mapping of Magnetic Fields in Star-forming Regions. I. W51 e1/e2 Molecular Cores, Apj 561, 864 (2001).

[40] D.S. Wiebe, W.D. Watson, Irregular Magnetic Fields and the Far-Infrared Polarimetry of Dust Emission from Interstellar Clouds, ApJ 615, 300 (2004).

[41] D.S. Wiebe, W.D. Watson, Irregular Magnetic Fields in Interstellar Clouds and the Linear Polarization of Starlight, ApJL 549, L115 (2001).

[42] D.A. Schleuning, Far-Infrared and Submillimeter Polarization of OMC-1: Evidence for Magnetically Regulated Star Formation, ApJ 493, 811 (1998).

[43] R. Rao, R.M. Crutcher, R.L. Plambeck, M.C.H. Wright, High-Resolution Millimeter-Wave Mapping of Linearly Polarized Dust Emission: Magnetic Field Structure in Orion, ApJL 502, L75 (1998). 
[44] Y.-W. Tang, P.T.P. Ho, P.M. Koch, S. Guilloteau, A. Dutrey, Dust Continuum and Polarization from Envelope to Cores in Star Formation: A Case Study in the W51 North Region, ApJ 763, 135 (2013).

[45] R. Gehrz, Sources of Stardust in the Galaxy, in proceedings of Interstellar Dust, IAU Symp. No. 135, p. 445 (1989).

[46] A.P. Jones, A.G.G.M. Tielens, D.J. Hollenbach, Grain Shattering in Shocks: The Interstellar Grain Size Distribution, ApJ 469, 740 (1996).

[47] S. Zhukovska, Dust Origin in Late-Type Dwarf Galaxies: ISM Growth vs. Type II Supernovae, A\&A 562, A76 (2014).

[48] R. Kandori, K. Dobashi, H. Uehara, F. Sato, K. Yanagisawa,Grain Growth in the Dark Cloud L1251, AJ 126, 1888 (2003).

[49] Th.S. Allen, J.J. Prchlik, S.Th. Megeath, R.A. Gutermuth, J.L. Pipher, T. Naylor, R.D. Jeffries, An Anomalous Extinction Law in the Cep OB3b Young Cluster: Evidence for Dust Processing during Gas Dispersal, ApJ 786, 113 (2014).

[50] L. Campeggio, F. Strafella, B. Maiolo, D. Elia, S. Aiello, Total to Selective Extinction in the Dark Globule CB 107, ApJ 668, 316 (2007).

[51] J. Steinacker, M. Andersen, W.-F. Thi, R. Paladini, M. Juvela, A. Bacmann, V.-M. Pelkonen, L. Pagani, C. Lefèvre, Th. Henning, A. Noriega-Crespo, Grain Size Limits Derived from 3.6 mm and 4.5 $\mu m$ Coreshine, A\&A 582, A70 (2015).

[52] N.V. Voshchinnikov, Th. Henning, From Interstellar Abundances to Grain Composition: the Major Dust Constituents $\mathrm{Mg}$, Si, and Fe, A\&A 517, A45 (2010).

[53] E. Churchwell, M.S. Povich, D. Allen, M.G. Taylor, M.R. Meade, B.L. Babler, R. Indebetouw, C. Watson, B.A. Whitney, M.G. Wolfire, T.M. Bania, R.A. Benjamin, D.P. Clemens, M. Cohen, C.J. Cyganowski, J.M. Jackson, H.A. Kobulnicky, J.S. Mathis, E.P. Mercer, S.R. Stolovy, B. Uzpen, D.F. Watson, M.J. Wolff, The Bubbling Galactic Disk, ApJ 649, 759 (2006).

[54] R.J. Simpson, M.S. Povich, S. Kendrew, C.J. Lintott, E. Bressert, K. Arvidsson, C. Cyganowski, S. Maddison, K. Schawinski, R. Sherman, A.M. Smith, G. Wolf-Chase, The Milky Way Project First Data Release: a Bubblier Galactic Disc, MNRAS 424, 2442 (2012.)

[55] L. Deharveng, F. Schuller, L.D. Anderson, A. Zavagno, F. Wyrowski, K.M. Menten, L. Bronfman, L. Testi, C.M. Walmsley, M. Wienen, A Gallery of Bubbles. The Nature of the Bubbles Observed by Spitzer and what ATLASGAL Tells us about the Surrounding Neutral Material, A\&A 523, A6 (2010).

[56] Ya.N. Pavlyuchenkov, M.S. Kirsanova, D.S. Wiebe, Infrared Emission and the Destruction of Dust in HII Regions, Astronomy Reports 57, 573 (2013).

[57] D.S. Wiebe, M.S. Khramtsova, O.V. Egorov, T.A. Lozinskaya, Dust Evolution in the Dwarf Galaxy Holmberg II, Astronomy Letters 40, 278 (2014).

[58] M.S. Khramtsova, D.S. Wiebe, T.A. Lozinskaya, O.V. Egorov, Optical and Infrared Emission of HII Complexes as a Clue to the PAH Life Cycle, MNRAS 444, 757 (2014).

[59] A.P. Jones, L. Fanciullo, M. Köhler, L. Verstraete, V. Guillet, M. Bocchio, N. Ysard, The Evolution of Amorphous Hydrocarbons in the ISM: Dust Modelling from a new Vantage Point, A\&A 558, A62 (2013).

[60] M.S. Murga, S.A. Khoperskov, D.S. Wiebe, Restructuring and Destruction of Hydrocarbon Dust in the Interstellar Medium, Astronomy Reports 60, 233 (2016). 
[61] M.S. Murga, S.A. Khoperskov, D.S. Wiebe, The Evolution of Hydrocarbon Dust Grains in the Interstellar Medium and its Influence on the Infrared Spectra of Dust, Astronomy Reports 60, 669 (2016). 